\title{
First-episode psychosis in postpartum period: a
}

\section{review}

I. Pinto ${ }^{1}$, S. Garcia ${ }^{1}$, C. Ferreira ${ }^{1}$, M. Melo ${ }^{1}$, C. Pereira ${ }^{1}$, I. Caldas ${ }^{1}$, M. Bernardo ${ }^{2}$, M.J. Avelino ${ }^{3}$

${ }^{1}$ Trainee of psychiatry in Centro Hospitalar Psiquiátrico de Lisboa

${ }^{2}$ Trainee of psychiatry in Hospital Garcia de Orta, Almada

${ }^{3}$ Psychiatrist in Centro Hospitalar Psiquiátrico de Lisboa

\section{Objectives}

Collect and evaluate the latest information on post partum psychosis focusing especially on the first episodes and their classification.

\section{Background and aims}

Postpartum psychosis is a rare (1 in 1000 childbirths) and severe disorder that usually occur within the first 4 weeks after childbirth. It can occur alone in the perinatal context but is often associated with mooddisorder diathesis. Postpartum psychosis usually happens in primiparity, if so, will increase the risk of happening on the following pregnancies.

\section{Materials and methods}

A literature research was carried out on first-episode psychosis and first-onset psychosis in postpartum period or pregnancy. We also used severe psychiatric illness in postpartum as key words.

\section{Results}

Recent studies question the doctrine that correlates postpartum psychosis with mood disorder.

Although the vast majority of episodes occur in women who are subsequently diagnosed with mood disorders, some cases occur as the first psychotic episode of a schizophrenic disorder. Our research also found cases of individuals who only had episodes in the post partum context and with some unique features that propose the possibility of this being a clinical condition that deserves an individualized classification.

\section{Conclusions}

These new insights into this type of episode create the need for further long term follow-up studies in order to better characterize this syndrome, consequently allowing a better follow-up of the mother as well as an adequate maintenance therapy. 